\title{
The implications of multidiscipline and globalization
}

\author{
Haemi Jee
}

The words 'globalization' and 'multidiscipline' have been in popping up everywhere as if they are the need-to-have words to express the envisioning identity of an entity. However, do we really know what it takes to be global and multidiscipline? Although we may naturally envision diversity and expansion through the terms, a pause may await for what and how for such envisions. The literary definition states that globalization is about different views, products, cultures, ideas, and beliefs interchanged through the process of international integration. The term multidiscipline is defined as composition or combination of several fields of expertise. The word multidiscipline can be further defined as redefining complex problems outside of normal boundaries to achieve a new consensual understanding. In combination, it's about acquiring a new found consensual agreement between the experts with different disciplines.

It sounds fascinatingly productive and innovative to be global and multidiscipline. However, there's an intricate issue that should be noted before initiating the globally multidiscipline integration. These two words suggest bringing dissimilar or even contracting perspectives with years of screened disciplines for unification and integration. Establishing a renowned discipline may be close to a religious experience. It may have taken centuries to define and redefine certain discipline through a series of extensively meticulous multiple processes of categorizing and contemplating for its unique identity. A strong sense of singularity or singleness must be rooted in each unique discipline to withstand any disputation. Scholastic disciplines are based on such history. Although open to new concepts and findings, most of the scholastic disciplines may not care for intrusion of the very foundation or root of the ideology.

It may be in the human nature to categorize and differentiate the unlikeness. Intellectual maturation process is mostly based on identifying and debating on the difference. Scholastic minds are trained for such criticism and debates. How can such critical minds from drastically different perspectives integrate to find a commonly agreeable solution? There may be a need to prepare the critical minds for an intellectual integration.

The scientific communities have been trying to prepare the critical minds for such divert integration in recent years. Instead of rooting for solitude, the scientific communities have been offering a common ground to listen, debate, accept, and understand doctrines of different perspectives. Instead of forcing to confirm to a unity, a natural transition based on patience and time must be offered to let down the guards and accept the difference. Most of the judgmental bias or aversions begin as misinformed or lack of understanding of different stands. True acceptance cannot be ordered or forced but through personal experience and understanding.

A journal striving to be a ground for globalization of multidiscipline aspects needs to prepare itself to offer the critical minds for such natural transition. The need of all corners must be sought and considered so that all scholastic stands see each other with equality. It must allow adequate time for different perspectives to take in each other and understand where the others are coming from. It may seem complicated to make the critical minds to accept different stands and perspectives. However, it's not about making or manipulating the critical minds but showing the scholars the rational reasons to derive to a new solution. Rational inference is the very foundation of the critical thinking.

Globalization of multidisciplinary perspectives is a natural course of scientific evolution. It certainly is not the only course but one of the multiple paths necessary to strengthen the scientif-

This is an Open Access article distributed under the terms of the Creative Commons Attribution Non-Commercial License (http://creativecommons.org/licenses/by-nc/3.0/) which permits unrestricted non-commercial use, distribution, and reproduction in any medium, provided the original work is properly cited. 
ic findings necessary to improve the quality of lives. It may be an unavoidable course of nature. The overflow of information these days overwhelms to dilute the quality of information. It may be a natural flow of the era to select the right puzzles to complete the whole picture by allowing a controlled integrative ground for the critical minds to share their expertise. The rightful question may be to ask if the interactive ground is rightfully prepared for such puzzle sharing. The corresponding question may be what would be the rightful preparation. It may be very unique from one entity to another - integrating different disciplines, cultures, beliefs, or perspectives may not work a fixed type of solution but customized case-by-case adjustments.

The Journal of Exercise Rehabilitation (JER) has long been contemplating to organize such integrative venue for the scholars specializing in different sectors of exercise and medicine. The area that JER covers may seem comprehensive but the ultimate goal is to acquire more advance approach to support each discipline. The journal has been carefully laying the ground with all corners considered for the scholastic minds with different stands to come together. So far, without an unruly conflict or straggling, JER has managed to grow and expand from a multidisciplinary to a globally integrative journal. The journal's patience and consideration for all unique aspects has allowed understanding and acceptance by the scholars with different backgrounds. The critical scholars have accepted one another and contemplated integrative collaboration between different scholastic disciplines. The top minds unceasingly said that to stand is to fall behind. JER should continue its position as an integrative venue and expand to reach greater audience and debaters for further integration and new solutions.

Department of Sports and Health Care, Namseoul University, 91 Daehak-ro Seonghwan-eup, Sebuk-gu, Cheonan 31020, Korea E-mail:wlgPal9@gmail.com 